\title{
Effects of Eight-Week Resistance Training Program in Men With Multiple Sclerosis
}

\author{
Mahbubeh Moradi ${ }^{1,2}$; Mohammad Ali Sahraian ${ }^{1,3}$; Aida Aghsaie ${ }^{1,3} ;$ Mohammad Reza Kordi $^{2}$; \\ Alipasha Meysamie ${ }^{4}$; Maryam Abolhasani ${ }^{5,6,}{ }^{*}$ Vahid Sobhani $^{7}$ \\ ${ }^{1}$ MS Research Center, Neuroscience Institute, Tehran University of Medical Sciences, Tehran, IR Iran \\ ${ }^{2}$ Department of Sports Physiology, Faculty of Physical Education and Sport Sciences, University of Tehran, Tehran, IR Iran \\ ${ }^{3}$ Sina MS Research Center, Department of Neurology, Sina Hospital, Tehran University of Medical Sciences, Tehran, IR Iran \\ 4 Department of Community and Preventive Medicine, School of Medicine, Tehran University of Medical Sciences, Tehran, IR Iran \\ 5 Sports Medicine Research Center, Neuroscience Institute, Tehran University of Medical Sciences, Tehran, IR Iran \\ 6 Department of Sports Medicine, Sina Hospital, Tehran University of Medical Sciences, Tehran, IR Iran \\ ${ }^{7}$ Exercise Physiology Research Center, Baqyatallah University of Medical Sciences, Tehran, IR Iran \\ ${ }^{*}$ Corresponding author: Maryam Abolhasani, Sports Medicine Research Center, Neuroscience Institute, Tehran University of Medical Sciences, Tehran, IR Iran. Tel: +98-2188630228, \\ Fax: +98-2166348570, E-mail: Dr_m_abolhasani@yahoo.com
}

Received: August 17, 2014; Revised: November 8, 2014; Accepted: November 20, 2014

Background: Muscle weakness and fatigue contribute to the reduction of daily activity in multiple sclerosis (MS) patients. Therapeutic strategies to promote improvements in muscle strength and endurance are limited in individuals with MS. Some evidence showed that exercise may improve and affect different aspects of the disease including quality of life, fatigue, motor, and cognitive functions.

Objectives: To investigate the value of resistance training of moderate to high intensity on motor function, muscle strength, balance and perceived disability in male patients with multiple sclerosis compared to a control group.

Patients and Methods: 20 male patients with MS (mean \pm SD, age: $34.05 \pm 7.8$ y; Expanded Disability Status Scale (EDSS): $2.94 \pm 1.5$ ) were recruited and randomized either to the exercise (E) or control group (C). Group E participated in a three-time weekly individualized progressive resistance-training program (both upper and lower extremities) for eight weeks, while group C was advised not to change their physical activity habits. All initial measures (including EDSS, balance, muscle strength, and functional mobility) were re-evaluated at the end of the program.

Results: Two patients of group E left the program. The other eight subjects completed the program with no MS-related exacerbations/ complications. There was a significant change in 2 of 3 aspects of ambulatory function [Three minutes step test $(\mathrm{P}=0.001)$, Timed Up and Go test $(\mathrm{P}=0.009)]$, muscle strength $(\mathrm{P}=0.000)$, and $\mathrm{EDSS}(\mathrm{P}=0.014)$. Comparing the two groups, we did not observe any significant change in "Balance" $(\mathrm{P}=0.407)$.

Conclusions: The resistance training of moderate to high intensity was well-tolerated in MS patients and may be an effective intervention for improving muscle strength, functional ability and EDSS-based disease severity.

Keywords: Multiple Sclerosis; Resistance Training; Muscle Strength; Motor Activity

\section{Background}

Multiple sclerosis (MS) is a chronic demyelinating disease of the central nervous system that usually affects young adults and may lead to physical disability due to motor or sensory dysfunctions. It may also lead to problems with balance, coordination, postural control, and walking mechanics (gait). Muscle weakness and fatigue contribute to the reduction of daily activity in MS patients. Inactivity further compromises muscle function, ambulatory ability, and thus physical fitness (1). Therapeutic strategies to promote improvements in muscle strength and endurance are limited in individuals with MS (2). Although exercise is one of these strategies, for many years, participating in physical exercise was a controversial issue for the patients. This was because of the nature of the disease, thermosensitivity and easy fati- gability reported in such cases. Over $80 \%$ of the patients have been noted to develop increased neurologic signs as a result of a rise in core temperature (3). Fatigue resulting from central causes or secondary to muscle disuse and inactivity is the most common symptom experienced by patients $(4,5)$. Unlike central nervous system involvement, fatigue due to inactivity may be improved by an exercise program (4). Both causes may influence the ability of the patients to exercise, and may therefore limit their ability to benefit from exercise programs, particularly if they require vigorous training. It has recently been shown that post-exercise worsening of the number and/ or intensity of sensory symptoms, experienced by more than $40 \%$ of MS patients is temporal, and will be normalized within half an hour after exercise cessation in most

Copyright (c) 2015, Sports Medicine Research Center. This is an open-access article distributed under the terms of the Creative Commons Attribution-NonCommercial 4.0 International License (http://creativecommons.org/licenses/by-nc/4.0/) which permits copy and redistribute the material just in noncommercial usages, provided the original work is properly cited. 
cases (6). During the last decade, an increasing number of evidence showed that exercise may improve and affect different aspects of the disease including quality of life, fatigue, motor and cognitive functions. Various training exercises, such as resistance, endurance training, and combined training are known to target different areas of the physiologic profile in multiple sclerosis patients with mild to moderate disabilities (7). Previous studies on mild, nonsupervised progressive resistance training (PRT) reported an improvement in muscle strength after intervention. However, the effect of resistance training on functional capacity in MS remains unclear, and therefore, controlled studies of supervised and intense PRT of the upper and lower extremities on muscle strength and functional capacity in MS patients are needed $(7,8)$.

\section{Objectives}

The purpose of this study was to evaluate the effectiveness of 8-week progressive resistance training of upper and lower body muscles on motor function, muscle strength, balance and perceived disability in male MS patients compared to a control group.

\section{Patients and Methods}

Twenty MS male patients with the mean age of $34.05 \mathrm{y}$, mean BMI of 22/40 kg and Expanded Disability Status Scale (EDSS) (9) ranging from 1 to 6 from Iranian MS Society were recruited for this study. The inclusion criteria were, diagnosis of MS according to the revised Mc Donald criteria (10), 1.0-6.0 EDSS score, the age between 20 and 55 years, no history of cardiovascular or any other systemic disease, no history of relapse during the month before entering the study and non-participant in regular exercise at least 30 minutes per day for the previous three months. The participants completed an informed consent approved by the local ethical committee before enrollment; then, the patients were randomized either to the exercise (group E) or control group (group C) according to random digit by a table of random numbers. If the patients could not participate in more than one training session with any reason or had are lapse and exacerbation of the symptoms, they were excluded from the study. It should be noted that there was no intervention in the drug regimen of the patients and they were allowed to use their medications including disease modifying therapies.

\subsection{Study Design}

The duration of the study was 12 weeks. Participant screening, study orientation and baseline measurements of lower and upper extremity muscle strength, walking speed, stepping rate, balance and EDSS were all performed during the first two weeks. During the next eight weeks, the exercise group participated in a three-time weekly resistance-training program with 24-hour rest between consecutive exercise sessions, while the control group was advised not to change their physical activity habits during the same period. All initial measures (including EDSS, balance, muscle strength, and functional mobility) were re-evaluated during the final two weeks.

\subsection{Ambulatory Function}

Three measures of ambulatory function were assessed prior to and following the eight-week resistance-training program. The first measure consisted of the 10-meter timed walk test (10TW) (11). The subjects performed the walk test from a still start behind a starting line. They were asked to walk as quickly and safely as possible. Time was recorded from the point the "go" command was let out until the subject passed the finish line. The test was performed three consecutive times with five recovery minutes between trials. The mean time of the 3 trials was included in the analysis (11). The second measure was a three minute step test. The subjects were asked to step up onto a platform $15.2 \mathrm{~cm}$ ( 6 inch) above the floor with both legs as many times as possible in a 3-min period and the total number of steps was recorded. The subjects were provided with any assistance necessary to complete the step test. The maximum number of steps completed in three minutes was measured and recorded (11). The last measure to evaluate functional mobility was "Timed Up and Go" test. In this test, the time taken by a person to stand up from a standard arm chair, walk a distance of 3 $\mathrm{m}$, turn, walk back to the chair, and sit down again was measured (12). This test was performed 2 times, and the best score was used in the analysis. All these tests have been widely administered on adults in neurorehabilitation and have been determined to be reliable and valid indices of functional mobility (11).

\subsection{Muscle Strength Testing}

According to ACSM's (American College of Sports Medicine) advice, for maximal strength and endurance improvement, the resistance should be at an individual's 8 to 12 repetition maximum (RM) so that he is at or near maximal exertion at the end of the repetitions.

One repetition maximum ( $1 \mathrm{RM}$ ) is predicted using the following Equation 1(13):

\section{(1) $1 \mathrm{RM}=$ weight lifted $/ 1.0-($ number of lifts $\times 0.025)$}

Lower and upper limb muscle strength was evaluated by trial and error using the weight machines. A suitable weight machine was used for determining 1 RM of each major muscle group: seated rowing (for dorsal chest muscles), chest press (for triceps and anterior chest muscles), leg extension (for leg extensors) and leg press (for leg flexors) (14). For each exercise, the patients were asked to perform the task with the heaviest weight they could raise without getting into trouble. And they were asked to repeat it as many times as they could. Finally, the weight which could be lifted for 6 - 10 repetitions was chosen. The weight was lifted and $1 \mathrm{RM}$ was determined for 
each patient calculating the number of lifts in the above Equation 1 (13). The subjects completed a standardized warm-up prior to strength testing.

\subsection{Balance}

Balance was measured using the Flamingo Stand test. In this test, the free leg is flexed at the knee and the foot of this leg is held close to the buttocks while balancing on the preferred leg. The stopwatch was started as the subject raised his free leg from the floor. The stopwatch was stopped if the person lost balance (either by falling off the beam or letting goes off the foot being held). The test was performed 2 times, and the longest time was registered as the person's measure for balance (15).

\subsection{Resistance Training Protocol}

After group assignment and before the training program, the exercise group participated in 6 instructional sessions. The sessions were designed to teach the correct form of each exercise. The sessions included instructions on warm-up stretches, resistance training exercises, and cool-down activities. Instructions and clues were given to remind participants of the correct positioning and form during the exercise. The sessions were performed three times a week, on alternating days, for two weeks. After the two-week instructional phase, the subjects completed a supervised resistance training (three times weekly) program for eight consecutive weeks. As said before, 1 RM was determined for each participant regarding the results of baseline strength testing. The findings were used to estimate the resistance load during training. The training protocol was adopted from ACSM's resistance-training guidelines and recognized criteria for load assignment in older/ disabled persons (13). Each participant performed warm up exercises for 5 - 10 minutes before strength training on a stationary bicycle or treadmill followed by stretching appropriate muscle groups. At the end of every session, cooling down exercises was performed for 5 - 10 minutes. Conventional weight machines were used for upper and lower body exercises including seated rowing, chest press, leg extension and leg press $(13,14)$. During the first week of training, the subjects performed one set of 6 - 10 repetitions at $50 \%$ of $1 \mathrm{RM}$ on each machine. During the second week, the subjects performed one set of $10-15$ repetitions at $60 \%$ 1 RM. During the third and fourth weeks, they completed one set of 10 - 15 repetitions at 70\% of RM. In subsequent sessions, the participants completed one set of 10 - 15 rep- etitions at $80 \%$ of 1 RM (Table 1 ). When subjects were able to complete 15 repetitions for any exercise, the resistance was increased by $2 \%$ to $5 \%$ in consecutive sessions. Exercises were performed at a self-selected, comfortable pace with at least 1 minute of rest between each. Training sessions did not exceed $30 \mathrm{~min} / \mathrm{session}$. During all strengthening exercises, a wall, a chair, or a stable structure was located on the left or right side of the subjects and they could grasp it if their balance was compromised. Because of variability in ability levels, the exercise programs were individualized so that each participant would be able to perform the exercises safely and effectively. Strength measures were reassessed following 8 weeks of resistance training.

\subsection{EDSS Assessment}

All patients referred to Sina MS clinic for EDSS evaluation. The assessment was performed by an expert neurologist in multiple sclerosis, who was blinded to the type of intervention, before and after the study program. The patients were asked not to inform the examining physician if they performed the exercise. The physician was also blinded for pre study EDSS while evaluating the patients for the second time to avoid any bias.

\subsection{Statistical Analysis}

Baseline characteristics between exercise and control groups were compared by Mann-Whitney U tests (because of small sample size and probability of abnormal distribution). Data is presented as mean \pm /standard deviation (SD) for pretesting and post testing sessions for the primary outcome measures except for EDSS and type of MS (which are shown as "median \pm SD"). Due to the small sample size, nonparametric Wilcoxon signed ranks tests were performed to determine if any changes occurred in any measure following eight weeks of strength training (comparing pretest-posttest in each group). To conclude if the intervention was successful through the time, a 2 $\times 2$ repeated-measures analysis of variance (ANOVA) was used to examine the group, time, and group by time interaction. It was done by "groups" (either E or C) as "between-subjects factor" and "time" (either pretest or posttest) as "within-subjects factor". The interaction was used to determine differences in the groups during the intervention (group by time). All tests were performed using the traditional level of significance $(P \leq 0.05)$.

All statistical analyses were done using SPSS, version 17.0, Chicago, Illinois.

\begin{tabular}{lcccc}
\hline Table 1. Resistance Training Program Plan & & & \\
\hline Week Variable & 1st Week & 2nd Week & 3rd and 4th Weeks & 5th - 8th Weeks \\
\hline Duration & 30 minutes & 30 minutes & 30 minutes & 30 minutes \\
Intensity & $50 \%$ of 1-RM & $60 \%$ of 1-RM & $70 \%$ of 1-RM & $80 \%$ of 1-RM \\
Repetition & $6-10$ & $10-15$ & $10-15$ & $10-15$ \\
Number of sets & 1 set & 1 set & 1 set & 1 set \\
\hline
\end{tabular}


Moradi Met al.

\section{Results}

Two subjects of the exercise group left the program in the first week for personal reasons. Their matched controls were also excluded in the final evaluation. The other eight subjects completed the 8-week resistance-training program (16 sessions) with no MS-related exacerbations reported. If any subject missed a session for personal reasons make up sessions were considered in the protocol. One subject reported mild muscle tenderness during the first 2 weeks of the training, but his symptoms resolved within 2 to 3 days. The baseline characteristics of the subjects are presented in Table 2.

\subsection{Ambulatory Function}

\subsubsection{0-Meter Timed Walk Test}

The average walking time to complete 10 meters at baseline $(8.3 \pm 4.5 \mathrm{~s})$ changed significantly following training (6.6 $\pm 2.0 \mathrm{~s}, \mathrm{P}=0.006)$. This measure was $(7.5 \pm 1.6 \mathrm{~s})$ at baseline and $(7.5 \pm 1.6 \mathrm{~s}, \mathrm{P}=0.199)$ after 8 weeks for the control group. Considering the time, the interaction was not significant. The group by time interaction was not statistically significant $(\mathrm{P}=0.083)$.

\subsubsection{Three-Minute Step Test}

Steps completed in three minutes increased significantly (35.5 \pm 20.3 steps at baseline and $48.8 \pm 25.3$ after training, $\mathrm{P}=0.006)$. Compared to the control group $(23.7 \pm 22.2$ steps at baseline and $19.8 \pm 22.7$ after 8 weeks, $\mathrm{P}=0.248$ ), the change was statistically significant $(P=0.002)$.

\subsubsection{Timed Up and Go Test}

The final results for the up and go test indicated that the 8 men in the exercise group decreased their time by $18.76 \%$ ( $9.9 \pm 2.1 \mathrm{~s}$ at baseline and $8.1 \pm 2.2 \mathrm{~s}$ after training, $\mathrm{P}=0.006$ ), while the control group showed no statistically significant decline $(10.5 \pm 2.9 \mathrm{~s}$ at pretest and $10.7 \pm 2.9 \mathrm{~s}$ at posttest, $\mathrm{P}$ $=0.444)$. The change between exercise and control groups was also statistically significant $(\mathrm{P}=0.009)$.

\subsection{Muscle Strength Testing}

As mentioned before, every exercise machine has special target muscles: seated rowing for dorsal chest muscles, chest press for triceps and anterior chest muscles, leg extension for leg extensors and leg press for leg flexors. After the intervention, the exercise group showed a significant difference in the strength of all the muscle groups.

\subsection{Seated Rowing}

There was a significant change in dorsal chest muscles strength following the eight-week program. The average load (per kilogram) to complete seated rowing test at baseline (115.0 $\pm 12.1 \mathrm{~kg})$ changed significantly following training $(150.3 \pm 16.8 \mathrm{~kg}, \mathrm{P}=0.006)$. This measure was
$(95.3 \pm 23.5 \mathrm{~kg})$ at baseline and $(91.0 \pm 21.1 \mathrm{~kg}, \mathrm{P}=0.040)$ after 8 weeks for the control group, which showed a statistically significant decline. The group by time interaction was also statistically significant $(\mathrm{P}=0.000)$.

\subsection{Chest Press}

The strength of "the triceps and anterior chest muscles" which were examined by chest press machine, indicated a significant improvement in the exercise group (54.2 $\pm 11.5 \mathrm{~kg}$ at baseline and $70.7 \pm 11.5 \mathrm{~kg}$ after training, $\mathrm{P}=$ 0.006), while no statistically significant decline (38.2 \pm $18.5 \mathrm{~kg}$ at pretest and $32.7 \pm 18.0 \mathrm{~kg}$ at posttest, $\mathrm{P}=0.072$ ) was observed in the control group. The change between exercise and control groups was statistically significant $(\mathrm{P}=0.000)$.

\subsection{Leg Extension}

There was a significant change in leg extensor muscles strength following the training program on leg extension machine. The average load (per kilogram) at baseline $(46.0 \pm 8.4 \mathrm{~kg})$ changed significantly following training (59.9 $\pm 11.6 \mathrm{~kg}, \mathrm{P}=0.006)$. This measure was $(26.7 \pm 8.3$ $\mathrm{kg})$ at pretest and $(22.9 \pm 9.3 \mathrm{~kg}, \mathrm{P}=0.021)$ posttest for the control group, which showed a statistically significant decline. The exercise and control groups showed a statistically significant change due to leg extensors strength following training program $(\mathrm{P}=0.000)$.

\subsection{Leg Press}

The strength of "leg flexors" examined by leg press machine indicated a significant improvement in the exercise group (115.9 $\pm 26.2 \mathrm{~kg}$ at baseline and $147.1 \pm 33.3 \mathrm{~kg}$ after training, $\mathrm{P}=0.006)$, while in the control group, a statistically significant decline $(99.5 \pm 37.8 \mathrm{~kg}$ at pretest and $87.8 \pm 39.5 \mathrm{~kg}$ at posttest, $\mathrm{P}=0.014)$ was observed. The change between exercise and control groups was statistically significant $(\mathrm{P}=0.000)$.

\subsection{Balance}

The final results of the balance test indicated that 8 men in the exercise group did not have a statistically significant improvement in their Flamingo stand test (23.9 \pm 26.0 before and $34.1 \pm 36.4$ after, $\mathrm{P}=0.118)$. Comparing to the results of the control group $(21.4 \pm 34.7 \mathrm{~s}$ before and by $21.6 \pm 33.5$ after, $\mathrm{P}=0.172)$ the intervention was not statistically successful $(\mathrm{P}=0.407)$.

\subsection{Disease Progression}

EDSS scores (median \pm SD) decreased from $3.0 \pm 1.6$ to $1.5 \pm 1.4$ after 8 weeks of strength training $(P=0.037)$. The EDSS scores of control group was $3.0 \pm 1.5$ and $3.7 \pm 1.5$ respectively before and after eight weeks $(\mathrm{P}=0.033)$. The change between exercise and control groups is statistically significant $(\mathrm{P}=0.014)$. Primary and secondary outcome measures were shown in Table 3. 
Moradi M et al.

\begin{tabular}{|c|c|c|c|}
\hline Variable & Exercise Group, $(n=8)$ & Control Group, $(\mathbf{n}=\mathbf{1 0})$ & P Value \\
\hline Age, $y$ & $34.38 \pm 11.07$ & $33.13 \pm 7.08$ & 0.792 \\
\hline Height, m & $1.75 \pm 0.05$ & $1.72 \pm 0.05$ & 0.342 \\
\hline Weight, kg & $68.06 \pm 11.13$ & $63.75 \pm 9.47$ & 0.430 \\
\hline BMI, $\mathrm{kg} / \mathrm{m}^{2}$ & $22.15 \pm 3.63$ & $21.42 \pm 3.60$ & 0.674 \\
\hline Years after first symptoms (min-max) & $9.38 \pm 4.24(3-15)$ & $9 \pm 5.09(2-16)$ & 0.916 \\
\hline Years after diagnosis (min-max) & $8.12 \pm 4.79(2-15)$ & $6.5 \pm 5.78(1-14)$ & 0.368 \\
\hline EDSS, median (min-max) & $3(1-6)$ & $3(1-5)$ & 0.958 \\
\hline Using disease-modifying drugs, No. (\%) & $6(75)$ & $6(75)$ & - \\
\hline $\mathbf{R R} / \mathrm{SP}$ & $5 / 3$ & $6 / 4$ & - \\
\hline 10 meter timed walk test & $8.34 \pm 4.47$ & $7.26 \pm 1.55$ & 0.52 \\
\hline 3 times step test & $35.50 \pm 20.29$ & $23.70 \pm 19.80$ & 0.28 \\
\hline Time Up and Go Test & $9.91 \pm 2.28$ & $10.42 \pm 3.31$ & 0.705 \\
\hline Chest press $1 \mathrm{RM}$ & $54.2 \pm 11.5$ & $38.2 \pm 18.5$ & 0.052 \\
\hline Seated rowing $1 \mathrm{RM}$ & $115.0 \pm 12.1$ & $95.3 \pm 23.5$ & 0.254 \\
\hline Leg extension $1 \mathrm{RM}$ & $46.0 \pm 8.4$ & $26.5 \pm 8.9$ & 0.001 \\
\hline Leg Press 1 RM & $115.2 \pm 26.2$ & $99.51 \pm 22.5$ & 0.262 \\
\hline Balance test & $23.9 \pm 26$ & $21.4 \pm 34.7$ & 0.537 \\
\hline
\end{tabular}

a Abbreviations: BMI, body mass index; EDSS, expanded disability status scale; RR, relapsing remitting MS; SP, secondary progressive MS.

$\mathrm{b}_{\text {Values are mean } \pm S D \text {, unless otherwise noted. }}$

Table 3. Primary and Secondary Outcome Measures ${ }^{\text {a }}$

\begin{tabular}{|c|c|c|c|c|c|c|c|c|}
\hline \multirow[t]{2}{*}{ Variables } & \multicolumn{3}{|c|}{ Exercise Group } & \multicolumn{3}{|c|}{ Control Group } & & \multirow[t]{2}{*}{ P Value $^{\mathrm{d}}$} \\
\hline & Pretest $^{b}$ & Posttest $^{b}$ & P Value $^{\mathrm{C}}$ & Pretest $^{b}$ & Posttest $^{b}$ & PValue $^{\mathrm{C}}$ & Mean difference (95\% CI) & \\
\hline \multicolumn{9}{|l|}{ Ambulatory Function } \\
\hline 10-meter timed walk test & $8.3 \pm 4.5$ & $6.6 \pm 2.0$ & 0.118 & $7.3 \pm 1.6$ & $7.4 \pm 1.6$ & 0.199 & $1.8(0.1-3.5)$ & 0.083 \\
\hline Three minutes step test & $35.5 \pm 20.3$ & $48.8 \pm 25.3$ & 0.006 & $23.7 \pm 19.8$ & $19.8 \pm 20.7$ & 0.248 & $17.2(7.2-27.1)$ & 0.002 \\
\hline Timed up and go test & $9.9 \pm 2.3$ & $8.1 \pm 2.2$ & 0.006 & $10.5 \pm 3.31$ & $10.7 \pm 3.27$ & 0.444 & $2.2(1.5-2 / 9)$ & 0.009 \\
\hline \multicolumn{9}{|l|}{ Strength } \\
\hline Seated rowing & $115.0 \pm 12.1$ & $150.3 \pm 16.8$ & 0.006 & $95.3 \pm 23.5$ & $91.0 \pm 21.1$ & 0.040 & $39.7(29.5-48.9)$ & 0.000 \\
\hline Chest press & $54.2 \pm 11.5$ & $70.7 \pm 11.5$ & 0.006 & $38.2 \pm 18.5$ & $32.7 \pm 18.0$ & 0.072 & $22.7(13.1-32.3)$ & 0.000 \\
\hline Leg extension & $46.0 \pm 8.4$ & $59.9 \pm 11.6$ & 0.006 & $26.7 \pm 8.9$ & $22.0 \pm 8.9$ & 0.021 & $18.50(12.8-24.4)$ & 0.000 \\
\hline Leg press & $115.2 \pm 26.2$ & $147.1 \pm 23.3$ & 0.006 & $99.5 \pm 22.5$ & $87.8 \pm 20.6$ & 0.014 & $47.0(32.4-61.6)$ & 0.000 \\
\hline \multicolumn{9}{|l|}{ Balance } \\
\hline Flamingo stand test & $23.9 \pm 26.0$ & $34.1 \pm 36.4$ & 0.118 & $21.4 \pm 34.7$ & $21.6 \pm 33.5$ & 0.172 & $10.3(4.6-16.5)$ & 0.407 \\
\hline EDSS & $3.0 \pm 1.6$ & $1.5 \pm 1.4$ & 0.037 & $3 \pm 1.5$ & $3.7 \pm 1.5$ & 0.033 & $1.8(0.7-3)$ & \\
\hline
\end{tabular}

a Abbreviation: CI, confidence interval.

d Of change between groups with "group by time interaction" (by repeated measure ANOVA).

$\mathrm{b}$ Pretest and posttest are "mean \pm SD" except for EDSS which is "median \pm SD".

${ }^{\mathrm{C}}$ Of change between pretest and posttest (by Wilcoxon Signed Rank Test).

\section{Discussion}

Based on the existing literature, it seems that there is no doubt in positive effects of exercise programs on improving body function, mobility $(16,17)$ and quality of life (1) in patients with MS. Albeit there are still unanswered questions in advising widespread high intensity resistance exercise which needs high quality randomized 
Moradi Met al.

trials $(1,18)$. The main concern is designing the training to maximize benefits without any detrimental effects on the course of the disease. The present study demonstrated that supervised progressive resistance training of both upper and lower extremities can improve both muscle strength and ambulatory function in patients with mild to moderate MS while balance did not improve compared to the control group. Neither injuries nor relapses have been reported due to the present program load. Muscular strength of all muscle groups improved significantly in response to resistance training program which is in agreement with findings of previous studies $(8,15,19-21)$. In the studies of Broekmans et al. (22) and White et al. (20) which are comparable with our study (both using standard ACSM based training), the effects of muscle strength improvement (9\% and $7.4 \%$, respectively) were small compared to healthy individuals (15 $30 \%$ (22). In our study, muscle strength improvement of the subjects was similar to healthy subjects (27-30\%). The reason can be explained in the intensity of the protocol we used because we used progressive resistance training protocol with progression from moderate to high intensity. White et al. (20) started their program with 8-10 RM at 50\% and increased it up to $10-15$ RM at 70\%. Similarly, Broekmans et al. (22) started their program with 10RM at $50-60 \%$ and increased it up to 2 sets of 10 RM at $60 \%$. We gradually increased the intensity of the training up to 10 RM at $80 \%$. As mentioned before, it was well tolerated by the patients. The mentioned studies only targeted the lower limbs; Broekmans' (22) study was done unilaterally on only a single lower limb, indeed. To the best of our knowledge, there are a small number of studies targeting the upper extremities $(15,22)$, perhaps because the disease usually affects the legs sooner. Our program was as effective in upper extremities as lower ones. Another notable point in the results of the present study is the significant change in patients' EDSS $(\mathrm{P}=0.014)$ compared to the control group, which has not been achieved in previous studies $(8,20,22)$. Different studies have used different modalities (22) to perform resistance training program, there are few studies which used the standardized ACSM guidelines $(20,22)$. We used standardized ACSM's guideline for resistance training and assessment of older/disabled individuals and we individualized the resistance program according to baseline assessments. It seems that individualization is a good and successful way for strength training in multiple sclerosis patients. The existing literature also differs with regard to duration of intervention. It is variable from 2 weeks to 20 weeks for supervised non home-based programs. In the study of Broekmans et al. (22) which lasted for 20 weeks, muscle strength improvement reached a plateau in the first half of the study, and did not change during the second 10 weeks. But since the study didn't have a control group, it is unclear whether an MS-specific mechanism prevented further improvement or there is a plateau in training process like the healthy individuals (22). In our study we didn't reach a plateau during the strength training in exercise group. This can be because of progressive protocol and individualization the program for each patient. Another variable evaluated in the current study was ambulatory function, which was improved in all 3 tests done (10-meter timed walk test, TUG (Timed Up and GO) and 3-minute step test). The improvements observed in last two parameters were significantly compared to the control group. As there are various tests applied for evaluating mobility, the findings of previous studies are controversial $(9,19,22,23)$. 10-meter walk test improved in Cakt et al. (23) and Dalgas et al. (8) studies, which are in contrast with our findings. TUG improved in the study of Cakt et al. and supports our findings, while it remained unchanged in Broekmans (22) and DeBolt (19) studies. 3-minute step test was improved in Gutierrez et al.'s study (21), an 8 -week supervised resistance program, the same as ours. Snook et al. (17) meta-analysis on articles published from 1960 to November 2007 supports a small improvement in mobility due to exercise training in MS patients which is in agreement with findings of the current study. The last parameter evaluated in the study was balance, which neither improved in group E compared to group $\mathrm{C}$ nor before and after the training in group $\mathrm{E}$. The results mirror data from previous studies $(15,19,24)$. It necessitates specific balance training besides resistance programs. Cakt et al. (23) combined resistance and balance training which resulted in improved balance, that supports the idea. Our findings, especially in regard to muscle strength, have clinical importance, since the impaired muscle adaptation ability was underestimated in previous studies. Applying low to moderately intense regimens influenced the results of the previous studies, coming to the conclusion that exercise has a small effect on muscle strength. Current results may be a supportive evidence for the hypothesis that weak muscles disability in MS is more probably disuse-associated (22) and it responds to training programs as well as healthy individuals. However, it should be reproduced with a larger sample size.

In fact, improved muscle strength as a potential predictor of ambulatory function (22) explains current regimen success in improving mobility. The present study has several limitations. Firstly, the small sample size and its gender-based selection may influence coming to an absolute deduction. Secondly, no dynamometer calculation has been used in the present study, and all the improvements are based on the upgoing trend of the loads the participants could handle while practicing. We didn't use intention to treat in our analysis because of the lack of some following data from two missed cases in exercise group. This is another limitation of our study. Some strengths of our study are adherence to ACSM's resistance-training guidelines and recognized criteria for load assignment in older/disabled person's guideline for strength training, strength training of upper and lower extremity together along with assessment of $1 \mathrm{RM}$ of targeted group muscles 
and 8 weeks supervised strength training. In conclusion, our study shows that an individualized ACSM-based progressive resistance training program can improve muscle strength and ambulatory function in MS patients. It also has a significant positive effect on EDSS, but may not improve balance. These effects are obtained in an 8-week regimen of resistance training of moderate to high intensity. Future studies with larger sample sizes, involving both sexes with time extension are suggested to shed more light on these important topics.

\section{Acknowledgements}

The others would like to thank Development and Research center of Sina Hospital and Mrs Poormand for translation in English.

\section{Funding/Support}

The funding for the project was provided by Tehran University, Faculty of Physical Education and Sport Sciences, Department of Sports Physiology.

\section{References}

1. Motl RW, Gosney JL. Effect of exercise training on quality of life in multiple sclerosis: a meta-analysis. Mult Scler. 2008;14(1):129-35.

2. Frzovic D, Morris ME, Vowels L. Clinical tests of standing balance: performance of persons with multiple sclerosis. Arch Phys Med Rehabil. 2000;81(2):215-21.

3. Guthrie TC, Nelson DA. Influence of temperature changes on multiple sclerosis: critical review of mechanisms and research potential. J Neurol Sci.1995;129(1):1-8.

4. Karpatkin HI. Multiple sclerosis and exercise: a review of the evidence. Int J MS Care. 2005;7(2):36-41.

5. Stuifbergen AK. Physical activity and perceived health status in persons with multiple sclerosis. J Neurosci Nurs. 1997;29(4):238-43.

6. Smith RM, Adeney-Steel M, Fulcher G, Longley WA. Symptom change with exercise is a temporary phenomenon for people with multiple sclerosis. Arch Phys Med Rehabil. 2006;87(5):723-7.

7. Dalgas U, Stenager E, Ingemann-Hansen T. Multiple sclerosis and physical exercise: recommendations for the application of resistance-, endurance- and combined training. Mult Scler. 2008;14(1):35-53.

8. Dalgas U, Stenager E, Jakobsen J, Petersen T, Hansen HJ, Knudsen C, et al. Resistance training improves muscle strength and functional capacity in multiple sclerosis. Neurology. 2009;73(18):1478-84.

9. Kurtzke JF. Rating neurologic impairment in multiple scle- rosis an expanded disability status scale (EDSS). Neurology 1983;33(11):1444.

10. Polman CH, Reingold SC, Banwell B, Clanet M, Cohen JA, Filippi $\mathrm{M}$, et al. Diagnostic criteria for multiple sclerosis: 2010 revisions to the McDonald criteria. Ann Neurol. 2011;69(2):292-302.

11. Rossier P, Wade DT. Validity and reliability comparison of 4 mobility measures in patients presenting with neurologic impairment. Arch Phys Med Rehabil. 2001;82(1):9-13.

12. Podsiadlo D, Richardson S. The timed "Up \& Go": a test of basic functional mobility for frail elderly persons. J Am Geriatr Soc. 1991;39(2):142-8.

13. Balady GJ, Berra KA, Golding LA. ACSM's Guidelines for Exercise Testing and Prescription. 9th edPhiladelphia: Lippincott Williams \& Wilkins; 2010

14. Learmonth YC, Paul L, McFadyen AK, Mattison P, Miller L. Reliability and clinical significance of mobility and balance assessments in multiple sclerosis. Int J Rehabil Res. 2012;35(1):69-74.

15. Ayan Perez C, Martin Sanchez V, De Souza Teixeira F, De Paz Fernandez JA. Effects of a resistance training program in multiple sclerosis Spanish patients: a pilot study. J Sport Rehabil. 2007;16(2):143-53.

16. Garrett M, Coote S. Multiple sclerosis and exercise in people with minimal gait impairment-a review. Phys Ther Rev. 2009; 14(3):169-80.

17. Snook EM, Motl RW. Effect of exercise training on walking mobility in multiple sclerosis: a meta-analysis. Neurorehabil Neural Repair. 2009;23(2):108-16.

18. Asano M, Dawes DJ, Arafah A, Moriello C, Mayo NE. What does a structured review of the effectiveness of exercise interventions for persons with multiple sclerosis tell us about the challenges of designing trials? Mult Scler. 2009;15(4):412-21.

19. DeBolt LS, McCubbin JA. The effects of home-based resistance exercise on balance, power, and mobility in adults with multiple sclerosis. Arch Phys Med Rehabil. 2004;85(2):290-7.

20. White LJ, McCoy SC, Castellano V, Gutierrez G, Stevens JE, Walter GA, et al. Resistance training improves strength and functional capacity in persons with multiple sclerosis. Mult Scler 2004;10(6):668-74.

21. Gutierrez GM, Chow JW, Tillman MD, McCoy SC, Castellano V, White LJ. Resistance training improves gait kinematics in persons with multiple sclerosis. Arch Phys Med Rehabil. 2005;86(9):1824-9.

22. Broekmans T, Roelants M, Feys P, Alders G, Gijbels D, Hanssen I, et al. Effects of long-term resistance training and simultaneous electro-stimulation on muscle strength and functional mobility in multiple sclerosis. Mult Scler. 2011;17(4):468-77.

23. Cakt BD, Nacir B, Genc H, Saracoglu M, Karagoz A, Erdem HR, et al Cycling progressive resistance training for people with multiple sclerosis: a randomized controlled study. Am J Phys Med Rehabil. 2010;89(6):446-57.

24. Romberg A, Virtanen A, Ruutiainen J, Aunola S, Karppi SL, Vaara $\mathrm{M}$, et al. Effects of a 6-month exercise program on patients with multiple sclerosis: a randomized study. Neurology. 2004;63(11):2034-8. 